\title{
Una mirada al desarrollo de aditivos reductores de viscosidad y sus aplicaciones en el transporte de crudos pesados
}

\section{An overview to development of viscosity reducer additives and its application in heavy crude oil transportation}

\section{Um olhar sobre o desenvolvimento de aditivos para redução da viscosidade e suas aplicações no transporte de petróleo pesado}

\author{
Sergio Arturo Remolina-Campos ${ }^{1}$; Jaime Espitia-Castellanos ${ }^{1}$; César Augusto Luna-Cáceres ${ }^{1 *}$; \\ Édgar Javier Patiño-Reyes ${ }^{2}$ \\ ${ }^{1}$ Universidad Pontificia Bolivariana Seccional Bucaramanga (UPB), km 7 vía Piedecuesta, \\ Bucaramanga, Colombia \\ ${ }^{2}$ Instituto Colombiano del Petróleo (ICP Ecopetrol S. A.), km 7 vía Piedecuesta, Bucaramanga, Colombia \\ *cesar.luna@upb.edu.co
}

\begin{abstract}
Resumen
La presente revisión aborda la problemática que presentan los crudos pesados debido a su alta viscosidad, lo cual dificulta su transporte por tubería y su directa relación con la fracción de asfaltenos, cuyo apilamiento genera cambios en la reología del crudo, altas caídas de presión y mayores requerimientos energéticos en el bombeo, e incluso taponamiento de tuberías. Las investigaciones reportadas se han enfocado en predecir y elucidar la composición estructural de los asfaltenos, de lo cual se resaltan modelos que describen a los asfaltenos como sistemas de anillos aromáticos policondensados, que pueden unirse por interacciones de Van der Waals formando agregados, o también como estructuras jerárquicas de sistemas de anillos de hidrocarburos aromáticos policíclicos (PAH), que finalizan en la formación de racimos (clúster). También se resalta el modelo termodinámico coloidal, el cual predice moléculas de asfaltenos suspendidas en una fase líquida, estabilizada por resinas adsorbidas en su superficie.
\end{abstract}

Una alternativa para disminuir la viscosidad de los crudos es la inclusión de aditivos, los cuales mediante interacciones moleculares apropiadas, generan la dispersión de agregados asfalténicos, reflejándose en la disminución de la viscosidad. Por ello a la hora de diseñar aditivos reductores de viscosidad pare crudos, es importante diseñar estructuras moleculares con determinados grupos funcionales asociados a aminas, amidas, alcoholes, ácidos, entre otros, que favorezcan interacciones que generen la dispersión de asfaltenos. Por ellos y con el fin de brindar un panorama amplio de esta línea de investigación tan extensa, se finaliza este documento con una revisión de algunos aditivos reductores de viscosidad, implementados por diferentes investigadores.

Palabras clave: Aditivo; Asfaltenos; Interacciones; Nanoagregados; Viscosidad; Transporte de Crudo.

\begin{abstract}
The present review is addressed the problem of heavy crude oil, due to its high viscosity, making it difficult to transport by pipeline and its direct relationship with the asphaltenes fraction, whose stacking generates changes in crude rheology, high pressure drops and energy requirements in pumping and
\end{abstract}


even plugging of pipes. The reported investigations have focused on predicting and elucidating the structural composition of asphaltenes, from which are highlighted the models that describe asphaltenes as polycondensed aromatic ring systems that can be bound by van der Waals interactions in aggregates, also as hierarchical structures of ring systems of polycyclic aromatic hydrocarbons (PAH), that ends in formation of bunches (clusters). The colloidal thermodynamic model is also highlighted, which predicts that the molecules of asphaltenes are suspended in a liquid phase, stabilized by resins adsorbed on its surface.

An alternative to reduce the viscosity of crudes is the inclusion of additives, which through appropriate molecular interactions generate the dispersion of asphaltenic aggregates, reflected in the decrease in viscosity. For them, when designing viscosity reducing additives for crude oils, it is important to design molecular structures with certain functional groups associated with amines, amides, alcohols, acids, among others, that favor interactions that generate asphaltene dispersion. Therefore, and in order to provide a broad overview of this line of research so extensive, this document is finalized with a review of some viscosity reducing additives implemented by different researchers.

Keywords: Additive; Asphaltenes; Interactions; Nanoaggregate; Viscosity; Transport.

\section{Resumo}

A presente revisão considera a problemática que apresentam os crus pesados, devido a sua alta viscosidade, dificultando o seu transporte pela tubulação e sua direita relação com a fracção de asfaltenos, cujo empilhamento gera câmbios na reologia do cru, altas perdas de pressão e maiores requerimentos energéticos no bombeamento, até o tamponamento da tubulação. As pesquisas reportadas têm se direcionado em predizer e elucidar a composição estrutural dos asfaltenos, ressaltando modelos que descrevem aos asfaltenos como sistemas de anéis aromáticos policondensados, que podem-se unir pelas interações de Van der Waals formando agregados, também como estruturas hierárquicas de sistemas de anéis de hidrocarbonetos aromáticos policíclicos (PAH), que finalizam na formação de clusters. Também se ressalta o modelo termodinâmico coloidal, o qual prediz moléculas de asfaltenos suspensas numa fase liquida, estabilizada pelas resinas adsorvidas na sua superfície.

Uma alternativa para reduzir a viscosidade dos crudes é a inclusão de aditivos, que através de interações moleculares apropriadas geram a dispersão de agregados asfaltênicos, refletidos na diminuição da viscosidade. Para eles, ao projetar aditivos redutores de viscosidade para óleos crus, é importante projetar estruturas moleculares com certos grupos funcionais associados a aminas, amidas, álcoois, ácidos, entre outros, que favoreçam interações que geram dispersão de asfaltenos. Portanto, e a fim de fornecer uma visão ampla desta linha de pesquisa tão extensa, este documento é finalizado com uma revisão de alguns aditivos redutores de viscosidade implementados por diferentes pesquisadores.

Palavras-chave: Aditivo; Asfaltenos; Nano-Cúmulos; Viscosidade; Transporte de Cru.

\section{Introducción}

A lo largo de los últimos años, se ha evidenciado una disminución de las reservas mundiales de crudos convencionales (ligeros e intermedios), y una constante necesidad de suplir la demanda energética actual y futura a nivel mundial [1-4]. Debido a esto, se ha incrementado significativamente el desarrollo de fuentes alternativas de energía renovable, sostenible y amigable con el medio ambiente, destacándose la solar térmica, solar fotovoltaica, eólica, geotérmica, nuclear e hidroeléctrica, entre otras.
Sin embargo, el uso de combustibles fósiles sigue siendo fundamental para la economía mundial [5], por lo que se estima que hasta 2040 el petróleo continuará siendo la mayor fuente de energía [6]. Esto se confirma con el escenario base que plantea la Agencia Internacional de Energía (International Energy Agency), donde enuncia que la demanda mundial de energía primaria debería verse incrementada en un $66 \%$ para el 2030, con una tasa de crecimiento de $1,7 \%$ anual [7]. Es pertinente mencionar que, para cumplir con estos requerimientos energéticos mundiales, los hidrocarburos no convencionales presentan un 
rol protagónico [7-10], ya que se estima que las reservas de crudos convencionales son alrededor del $30 \%$, mientras que los crudos pesados (10$\left.20^{\circ} \mathrm{API}\right)$ y los extra-pesados $\left(10^{\circ} \mathrm{API}\right.$ o menos) conforman aproximadamente el $40 \%$ de los recursos totales [11-13], con cantidades que exceden los trillones de barriles [14-16].

Es conocido que en muchos países como Rusia, Canadá, Venezuela y Colombia, existen grandes depósitos de hidrocarburos pesados [7, 17-19], los cuales presentan elevadas viscosidades que dificultan su extracción y transporte. Esto se refleja en los altos requerimientos energéticos para el bombeo, junto con grandes caídas de presión en las tuberías [20]. Durante décadas habían sido evadidas la investigación y las mejoras de los procesos asociados con crudos pesados y extrapesados, por el alto costo requerido para la producción de barriales; pero ahora, debido a la gran demanda y las bajas reservas de crudo liviano existentes, se hacen necesarias la investigación y la búsqueda de nuevas tecnologías que faciliten su exploración, extracción y transporte.

Otro punto crítico en el manejo de crudos pesados es el envío desde los centros de producción hasta los puntos de refinación o comercialización. Una solución inmediata para la industria son los medios de transporte motorizados, en su gran mayoría carrotanques. No obstante, se considera que el oleoducto es el más económico y eficiente medio de transporte de crudo y sus diferentes productos [21, 22]. Los crudos pesados/extra-pesados (bitúmenes) son más difíciles de producir y transportar, debido a la agregación de sus moléculas de asfaltenos [1, 23], que ocasionan cambios de permeabilidad y humectabilidad en la formación, así como aumento de la masa molecular, cambios en la viscosidad y taponamiento de tuberías [24]. A esto se le suma que los oleoductos existentes fueron diseñados para transportar crudo convencional mas no pesado y extrapesado. Por estos motivos, es importante desarrollar e implementar tecnologías alternativas que faciliten el transporte de crudos pesados y extrapesados, entre los cuales el diseño de aditivos de reductores de viscosidad (ARV) es una alternativa viable, con la cual se disminuye la viscosidad de los crudos, y se logran cumplir los estándares requeridos para su transporte por tubería diseñada para crudos convencionales. En el presente artículo se pretende mostrar avances en el uso de sustancias químicas como ARV, los cuales actúan como dispersantes de asfaltenos valiéndose de las interacciones entre sitios polares de las dos especies (Asfalteno y ARV) [25], a lo cual se le asocia el efecto de diminución de viscosidad de crudos pesados y extrapesados.

\section{Bases teóricas}

El petróleo crudo es una mezcla de hidrocarburos en coexistencia termodinámica de estado líquido, sólido y gaseoso, con un amplio rango de masas moleculares de naturaleza química diferente, cuyas propiedades dependen de su tipo y origen [21, 25]. La identificación estructural del petróleo se hace difícil debido a que es una mezcla compleja de compuestos orgánicos e inorgánicos con heteroátomos en su estructura, tales como el azufre, nitrógeno, oxígeno y trazas de elementos metálicos como el zinc, hierro, níquel, vanadio, entre otros.

Una alternativa para poder estudiar los crudos, especialmente las fracciones pesadas, es el análisis SARA, cuya técnica consiste en hacer pasar el crudo por una columna cromatográfica que se encarga de separar las fracciones según su polaridad. De este análisis se obtienen fracciones de cuatro grandes familias: Saturados, Aromáticos, Resinas y Asfaltenos, de cuyas letras iniciales se forma la sigla que identifica a esta técnica [19]. La fracción de saturados está conformada principalmente por moléculas de carácter no polar, la de aromáticos por estructuras polares con electrones pi deslocalizados entre estructuras cíclicas con enlaces simples y dobles alternados, mientras que los asfaltenos y resinas son compuestos con secciones moleculares polares producidas por heteroátomos [24, 26-28].

Los crudos pesados usualmente contienen hidrocarburos de mayor masa molecular, elevados niveles de heteroátomos como azufre, nitrógeno, oxígeno, metales, y, además, presentan los mayores contenidos de resinas y asfaltenos en comparación con los convencionales. Particularmente, los asfaltenos son la fracción más densa, y están compuestos estructuralmente por una sección de grupos polares (tendencia dada por presencia de heteroátomos principalmente) enlazados a anillos aromáticos policondensados, de los cuales se derivan cadenas alifáticas, las cuales varían en su longitud [7, 19].

Debido a un sinnúmero de factores, los asfaltenos son estructuras complejas de alta masa molar, cuya aromaticidad repercute en mayores viscosidades $y$, por ende, en una menor movilidad de la fase 
continua del crudo (resaltando que en el lenguaje de la industria del petróleo, la fase continua es la oleica) [12, 29, 30]. Un aspecto fundamental para resolver este problema adyacente a los crudos pesados es comprender los mecanismos de apilamiento de asfaltenos [27, 31] y las diferentes interacciones que se generan entre las fracciones del crudo. Al respecto, muchos investigadores han planteado diferentes modelos y teorías sobre la estructura de las fracciones pesadas del crudo, las cuales se discuten a continuación.

En la actualidad se ha mejorado significativamente la compleja labor de caracterización estructural de los componentes del petróleo, lo que conlleva a una mejora en la predicción de la estabilidad de los asfaltenos y de otras propiedades básicas moleculares. Esto es posible hoy gracias a los grandes avances en la espectroscopia, especialmente en la resonancia magnética nuclear, espectroscopía raman, imágenes moleculares [24] y la espectrometría de masas por resonancia de ciclotrones de transformada de Fourier (FT-ICR $M S)$, entre otras [11, 32-34]; con las cuales es posible elucidar estructuras químicas de hasta 100.000 componentes presentes en el crudo, siendo este un gran paso al entendimiento del comportamiento de las propiedades del crudo, a través de las interacciones de índole química.

Para elucidar el comportamiento de los asfaltenos en el crudo, se resalta el modelo planteado por Teh Fu Yen [32, 35], el cual se basa en resultados experimentales, con los cuales se pudieron analizar las fracciones más pesadas del crudo, y se lograron establecer las estructuras medias de resinas, asfaltenos y bitúmenes. Este es un modelo que jerarquiza las estructuras dentro del crudo, y es el primer modelo en utilizar el término 'micela' para los sistemas de anillos aromáticos fusionados de moléculas de asfaltenos en forma de apilamientos pequeños [36]. Yen planteó que las moléculas de asfaltenos pueden unirse en agregados cuando su concentración es suficientemente alta. Las moléculas se unen por interacciones de Van der Waals [32, 35].

Aunque el modelo de Yen tuvo limitaciones significativas, ha ayudado a proponer una serie de modelos [28, 37-43] que en la actualidad cobran relevancia en el estudio de los asfaltenos, y que permiten entender los fenómenos de agregación interfacial de estos [44]. Más adelante, Mullins y colaboradores rescatan la información dada por Yen, respecto a la estructura de los asfaltenos, y plantean que estas estructuras jerárquicas de sistemas de anillos de hidrocarburos aromáticos policíclicos (PAH) de tamaño moderado [34, 45], periféricamente sustituidos de alcanos, pueden agregarse y formar nanoagregados estabilizados periféricamente por hidrocarburos de tipo alcanos, con números de agregación de $\sim 6, y$, a su vez, estos nanoagregados pueden formar racimos (clúster) con números de agregación estimados de 8 [45$50]$, el cual se conoce como modelo modificado de Yen (modelo de Yen-Mullins) [36]. Este modelo suministra las bases para comprender la forma en que se dispersan los asfaltenos en el crudo, dependiente de la concentración, dde la densidad y de las fuerzas moleculares. Adicionalmente, enuncia cómo los cambios en temperatura, presión o concentración ocasionan desestabilización de los asfaltenos, al estar inmersos en las resinas, lo cual genera la precipitación de estos de manera irreversible.

Otro modelo interesante que cabe mencionar es el termodinámico coloidal [24, 51], el cual predice que las moléculas de asfaltenos se encuentran suspendidas en una fase líquida, estabilizada por resinas adsorbidas en su superficie. El modelo de Yen-Mullins suministra adecuada información para desarrollar la ecuación de estado de asfaltenos (Flory-Huggins-Zuo) [41, 51-53], que ha ayudado a tener un mejor panorama de la participación de los asfaltenos en las propiedades del crudo, lo que facilita, en cierta medida, la producción en yacimientos de crudos pesados y extrapesados [11].

También se ha sugerido que las micelas tienen un núcleo asfalténico con aromaticidad de alta masa molar [54], además de una capa exterior compuesta de moléculas más ligeras y menos aromáticas [24]. La verdadera naturaleza de las micelas, con un núcleo asfalténico en particular, ha sido siempre un cuestionamiento para los investigadores de asfaltenos. Una de las preguntas más frecuentes es si existen los asfaltenos como capas apiladas dentro de las micelas o como unidades moleculares individuales, estabilizadas por las moléculas de resinas [46, 55]. Adicionalmente, Masoumeh Mousavi y colaboradores, investigaron la correlación entre el número de capas de los asfaltenos y la estabilidad final del sistema [54], en un medio compuesto de resinas y asfaltenos. El estudio se basó en simulación molecular, en la cual se implementó la teoría del funcional de densidad (DFT). Los hallazgos cuantitativos corroboraron que el comportamiento coloidal del crudo se describe mejor por asociaciones asfalteno- 
asfalteno. Además, los cálculos muestran que las interacciones moleculares resina-asfalteno son más estables que las interacciones asfaltenoasfalteno cuando hay más resinas que asfaltenos en la micela. Esto concuerda con evidencias experimentales asociadas con la estabilidad coloidal de los residuos de petróleo, las cuales sugieren que la estabilidad se logra cuando la concentración de resina es mayor que la de asfaltenos.

Como la estabilización coloidal de los asfaltenos ha sido atribuida típicamente a las fracciones de resinas, es imprescindible comprender las interacciones resina-asfalteno [24, 56-58]. A su vez, es tradicionalmente aceptado que la viscosidad de las dispersiones coloidales es altamente influenciada por el tamaño de los coloides [58] y si se logra desestabilizar la estructura micelar, así como romper los aglomerados de asfaltenos y dispersar esos compuestos orgánicos pesados, se obtiene una reducción de la viscosidad [59, 60]. Un estudio que aborda la conexión existente entre la composición y caracterización molecular de resinas y el comportamiento de estabilidad de asfaltenos fue realizado por Valbuena y colaboradores [61]. Los autores analizaron resinas de tipo I (fracción que coprecipita con los asfaltenos en exceso de $n$-heptano y que puede ser removida mediante extracciones soxhlet) y tipo II (obtenidas a partir de los maltenos, mediante cromatografía de adsorción por columna) de cuatro crudos venezolanos de diferente estabilidad operacional, mediante diversas técnicas espectroscópicas, entre las que se destacan infrarrojo, resonancia magnética nuclear de $1 \mathrm{H}$ y $13 \mathrm{C}$, osmometría de presión de vapor y análisis orgánico elemental de $\mathrm{C}, \mathrm{N}, \mathrm{O}$ y S. Basados en los resultados, pudieron concluir que las resinas tipo I tienen más afinidad hacia los asfáltenos que las tipo II, debido a su gran aromaticidad y pequeño tamaño molecular; esta última característica da la capacidad de entrar en microporos para precipitar con los asfaltenos. Adicionalmente, determinaron la presencia de grupos ácidos y alto contenido de azufre en resinas provenientes de crudos estables, lo cual evidencia la posible estabilidad del crudo por la generación de puentes de hidrógeno realizados entre los hidrógenos que acompañan a los heteroátomos de las resinas ( $\mathrm{C}, \mathrm{N}, \mathrm{O}$ y S) y los centros electrodonadores de los asfaltenos [61]. La información espectroscópica recolectada indica que las resinas tipo II son las responsables de la dispersión de asfaltenos en la matriz del crudo, ya que la cantidad y longitud de sus sustituyentes alquílicos o nafténicos representan un impedimento estérico entre ellas, inhibiendo la agregación y posterior precipitación de los asfaltenos en el crudo [61].

Así mismo, estudios teóricos sugieren que las interacciones resina-asfalteno ocurren a través de una combinación de efectos electrostáticos y de dispersión; se sugieren interacciones entre orbitales $\sigma \circ \pi$ de las resinas con el enlace- $\pi$ de los asfaltenos [62]. Adicionalmente, se reportan orientaciones $\pi-\pi$ stacking (cara a cara) como la configuración preferida entre las moléculas de resinas y asfaltenos [63]. La similitud estructural entre estas moléculas (resinas con menor masa molecular) es una razón para investigar estas conformaciones como las geometrías más eficientes en las interacciones resina-asfalteno [54]. Esto da a pensar que las interacciones intermoleculares, debidas a la aromaticidad, juegan un papel importante en la disminución de la viscosidad, es decir, las interacciones $\pi-\pi$.

De esta manera se evidencia que, al comprender los fundamentos teóricos relacionados con la composición, estructura e interacciones entre las fracciones del crudo, se permite dilucidar sus efectos en las propiedades reológicas del crudo, las cuales han sido consideradas como parámetros fundamentales en la búsqueda de condiciones óptimas en la transferencia de fluidos de hidrocarburos [21]. Gran parte de las cualidades reológicas de los crudos se atribuyen a la fracción de asfaltenos, lo cual constituye uno de los principales problemas que afronta la industria petrolera [56, 61, 64, 65], y uno de los principales ejes del presente artículo.

Diversos estudios buscan encontrar técnicas útiles para reducir la caída de presión y la viscosidad de los crudos por medio de métodos como calentamiento, dilución, emulsiones de crudo en agua, flujo anular inverso y upgrading parcial, con el objetivo de mejorar las propiedades de flujo de los crudos [1, 7, 21, 19, 66-68]. Al respecto, el calentamiento tiene un efecto dramático sobre la viscosidad del crudo pesado, pero no logra alcanzar un nivel práctico a escala industrial [20]. El uso de diluyentes genera una reducción significativa de la viscosidad, pero requiere grandes volúmenes, lo que en ocasiones representa altos costos operativos asociados al acondicionamiento reológico del crudo a una determinada condición de viscosidad [59]. Resumiendo, a pesar de los esfuerzos por encontrar nuevas tecnologías, 
estas han resultado en un aumento de los costos de capital y operación, e incluso en limitaciones técnicas [22]; por ello, es necesario mejorar las tecnologías actuales, las cuales deben contemplar rigurosamente las características fisicoquímicas, la composición y procedencia del crudo, el mejoramiento de infraestructura para el transporte de crudos pesados y extrapesados, y garantizar la reducción de viscosidad, de forma rentable, por medio de sustancias que sean más eficientes al ser empleadas como mejoradores de flujo, agentes reductores se resistencia al arrastre, agentes reductores de viscosidad, entre otros [69]. Cabe resaltar que existen diversas formas para disminuir la viscosidad de crudos pesados y bitumen, tal como lo reporta Abarasi Hart, quien explica detalladamente el efecto sobre dicha propiedad al calentar, diluir y aditivar [70]. Es de resaltar que uno de los efectos que marca una diferencia significativa en el cambio de viscosidad es el incremento de la temperatura, con el cual se logra aumentar los modos vibracionales moleculares de los componentes del sistema. A partir de esto se provoca una mayor movilidad de la fase continua, que se evidencia en el aumento de la viscosidad. A pesar de proporcionar un aumento marcado, en términos económicos y prácticos, no es un proceso viable. Otra alternativa es adicionar solvente, el cual debe ser afín con la fase continua del crudo (fase que se caracteriza por su tendencia apolar), ya que el fenómeno que genera la alteración de dicha propiedad es la solvatación. Uno de los solventes mas empleados es la nafta [71], la cual se caracteriza por ser una mezcla de sustancias con bajo peso molecular y alta apolaridad; asimismo, el gas líquido licuado (GLP por sus siglas en inglés) es otra alternativa de dilución [72]. Esta técnica es una de las más empleadas en el transporte de crudos, ya que es eficiente y rentable, e incluso existen diversos planteamientos teóricos basados en regla de mezclas, que buscan predecir el cambio de viscosidad al mezclar dos corrientes con viscosidades y densidades diferentes, como lo resaltan los modelos de Walther, Shu, entre otros [73]. La implementación de esta técnica es eficiente hasta cierto punto, ya que la disminución de la viscosidad no presenta una tendencia lineal sino un decaimiento exponencial con respecto al volumen de disolvente; esto quiere decir que al comienzo de la dilución el efecto es muy marcado, pero al seguir diluyendo el efecto empieza a disminuir hasta llegar al punto en que grandes cantidades de disolvente no evidencian disminución de la viscosidad. En este punto se hacen importantes los aditivos reductores de viscosidad, ya que, como su nombre lo indica, al adicionar pequeñas cantidades de sustancia (concentraciones en ppm), se generan grandes cambios en la viscosidad, producto de las interacciones que genera con los componentes del crudo. Esta es una técnica de alta investigación y el eje central de este artículo.

Es claro que una tarea clave para el sostenimiento de la industria de hidrocarburos es encontrar métodos de transporte de crudos crudos pesados, que sean eficientes y rentables. Potencialmente, se ha identificado el uso de aditivos como un método capaz de mejorar parámetros reológicos claves como la viscosidad de los crudos [20, 21]. Estos compuestos buscan interactuar con los asfaltenos imitando las interacciones que se presentan en los agregados de asfaltenos, tales como: tipo $\pi-\pi$ stacking, puentes de hidrógeno, interacciones ácido-base, fuerzas de Van der Waals, complejos coordinados y, preferiblemente, un carácter ácido a causa del carácter básico de Lewis de los asfaltenos [60].

\section{Aditivos reductores de viscosidad}

En esta sección se tocarán tópicos importantes a la hora de diseñar aditivos reductores de viscosidad, tales como su estructura y los vehículos. Se resalta que encontrar moléculas específicas que funcionen como aditivos y como mecanismos de acción, es un tema muy amplio, ya que depende de la naturaleza del crudo que se va a tratar.

Típicamente, los aditivos reductores de viscosidad se conforman por la mezcla de disolvente e ingrediente activo, o compuesto de naturaleza similar a las resinas [57]. Esta similitud se basa en que las resinas, por ser moléculas anfifílicas, estabilizan los asfaltenos por peptización [7476]. Por ello, la estructura del ingrediente activo consta normalmente de una cabeza polar, que contiene grupos ácidos o básicos que se adhieren al núcleo de la micela, y una cadena alifática hidrocarbonada que propicia un efecto estético alrededor de la Micaela, y esto genera interacciones débiles tipo dipolo inducido [77]. El objetivo principal del aditivo es atravesar la zona malténica y llegar hasta la zona polar, para modificar las interacciones resina-asfaltenos de los crudos pesados, y así resaltar la importancia del carácter dual del compuesto. Cabe resaltar 
que un apropiado balance hidrofílico-lipofílico (HLB) es esencial para una adecuada actividad interfacial [78].

Aspectos como la naturaleza química y la estructura del tensoactivo son parámetros importantes en la efectividad de las interacciones compuesto-asfaltenos [79, 80]. Relacionado con ello, Östlund y colaboradores concluyen que anfifílos, con grupos básicos en su cabeza, como el amino, presentan la menor adsorción en asfaltenos, mientras que grupos de ácido sulfónico tendrían un profundo efecto en la estabilidad de los asfaltenos [77]. Los autores concluyen que la adsorción de los anfifílos es muy importante para la estabilizaciónde asfaltenos, y que ello depende, no solo de los grupos funcionales del anfifilo, sino de la estructura química de los asfaltenos, remarcando la importancia de conocer la caracterización de los crudos. Por otra parte, Chávez y colaboradores concluyen que un ácido de Lewis es necesario en un extremo de la estructura para interactuar con los asfaltenos, así como la presencia de un grupo hidroxilo propicia la formación de puentes de hidrógeno [74]. Siguiendo esta línea, Osamah A. Alomair y colaboradores [81] reportan que compuestos capaces de formar puentes de hidrógeno con los asfaltenos inhiben la interacción asfalteno-asfalteno [82].

Adicionalmente, en la literatura se encuentra reporte de la importancia de la presencia de un grupo funcional carboxílico en la estructura del compuesto [83], lo cual hace que este tenga una mejor afinidad con la superficie de los asfaltenos. Esto es un factor positivo en la dispersión y estabilización [77], puesto que los puentes de hidrógeno entre aditivos ácidos y los sitios básicos de los asfaltenos son más evidentes que los enlaces entre aditivos básicos con los sitios ácidos en los asfaltenos. Adicionalmente, sugieren que un compuesto con un grupo funcional polar (como el hidroxilo), enlazado a un anillo aromático, fortalece la atracción y crea interacciones estables т-т con los asfaltenos, lo cual resalta la importancia en la estabilización de los asfaltenos. También destacan que inhibidores con alta polaridad y composición aromática actúan de manera similar a las resinas naturales en su función de mantener en solución a los asfaltenos.

De manera similar, Hussein y colaboradores reportan que [84], compuestos con alta polaridad y probabilidad de generar puentes de hidrógeno, se espera que evidencien un incremento y aceleración del rompimiento de los conglomerados de asfaltenos, lo que podría significar una mayor reducción de la viscosidad. Por el contrario, Badger y colaboradores concluyen que un carácter polar demasiado fuerte del dispersante causa una mayor viscosidad en el crudo [59], puesto que se pueden causar interacciones dipolo-dipolo entre los mismos compuestos, lo que disminuye la atracción con los asfaltenos.

Una alta concentración del compuesto, además de poder saturar la mezcla y llevarla al punto en el que una mayor dosificación no varíe la viscosidad [21], también puede incidir en una mayor tendencia a autoasociarse, por encima de las interacciones inhibidor-asfalteno, tal como reporta Karambeigi [83]. Para contrarrestar este efecto de autoasociación, se ha observado que la preferencia en la interacción asfaltenocompuesto puede ser favorecida con un mayor tamaño molecular del compuesto, puesto que se obtiene una interacción tan fuerte, capaz de formar agrupaciones estables con los asfaltenos [74]. Sin embargo, ha de tenerse en cuenta que ciertos autores sugieren compuestos con máximo un anillo aromático en la estructura, con el ánimo de mantener un tamaño de molécula adecuado, que mejore la difusión a través de la matriz del crudo y la penetración en los aglomerados de asfaltenos $[59,84]$.

Además de considerar los aspectos estructurales del extremo polar del compuesto, el efecto de la longitud de la cadena carbonada también es un aspecto clave en el comportamiento reológico del crudo aditivado [62, 85-87]. Al respecto, se ha encontrado que una cadena alquílica más larga aumenta significativamente la inhibición al prevenir las interacciones del agregado compuestoasfalteno con otros agregados o asfaltenos; mientras que, con una cadena más corta, la formación de agregados no es evitada, pero sí es estabilizada [74]. Esta información sugiere que el balance hidrofílico-lipofílico del tensoactivo es determinante en el tamaño del agregado de asfaltenos [23]. También coincide en indicar que líquidos iónicos con cadenas entre $\mathrm{C} 8-\mathrm{C} 12$ son más efectivos en la reducción de la viscosidad, en comparación con cadenas más cortas (C2C6), debido a que las cadenas más largas son capaces de inhibir las interacciones $\pi$ - $\pi$ entre moléculas de asfaltenos mediante la creación de una interferencia estérica.

También es importante mencionar que, en el proceso de crear adecuadas interacciones compuesto-asfalteno es importante revisar las 
fuerzas electrostáticas, las cuales son un factor determinante durante el proceso de adsorción del inhibidor. En un estudio realizado, se muestra que la adsorción de sustancias químicas aniónicas en yacimientos es alta, debido a que la superficie está cargada positivamente de minerales carbonados [83]. En crudos colombianos, Patiño y colaboradores comprobaron que los asfaltenos del crudo colombiano Rubiales presentan un carácter aniónico [17], por lo que los autores encontraron que el uso de un tensoactivo catiónico establece un marcado efecto de dispersión; mientras que con el crudo colombiano San Fernando se evidenció un caso contrario, puesto que fue un anfifilo de carácter aniónico el que tuvo un efecto positivo dispersante, a diferencia del catiónico que resultó promoviendo la precipitación.

Otro aspecto a considerar en la búsqueda de un compuesto con habilidades dispersantes y estabilizadoras de asfaltenos es su solubilidad en los crudos pesados [59]. Por este motivo, generalmente se utilizan diversos disolventes como vehículo, para solvatar el dispersante o compuesto en el crudo, para llevarlo hasta las micelas de asfaltenos. Es de resaltar que el uso de disolventes aromáticos mantiene en solución a las resinas [88], además de brindar un control a la precipitación de asfaltenos [62], puesto que estos son capaces de interrumpir los puentes de hidrógeno y las interacciones intermoleculares $\pi$, lo que reduce el grado de agregación de los asfaltenos [82]. Esto genera una estabilización de las micelas de asfaltenos y a una inhibición del proceso de precipitación [57]. Por otro lado, el uso de disolventes polares, tales como alcoholes, puede disminuir el tamaño de los agregados de asfaltenos, debido a la habilidad de establecer puentes de hidrógeno [81].

Todo el conocimiento adquirido en torno a la química de los asfaltenos ha permitido que a lo largo de los últimos años se consideren múltiples compuestos dispersantes y estabilizadores de los asfaltenos, los cuales buscan asemejarse estructuralmente a las resinas naturales y generar las interacciones adecuadas en búsqueda de lograr una disminución de la viscosidad en crudos pesados, y de esta manera, facilitar su transporte por oleoducto. En la literatura, se encuentran alternativas para poder conseguir esto, entre las que se destaca la enunciada por Llanos y colaboradores [89], quienes presentaron un bioreductor de viscosidad (BRV) no iónico realizado a base de ésteres de aceites vegetales, cuya reducción de viscosidad es semejante a la alcanzada por los xilenos, con la ventaja de que el $B R V$ es un recurso renovable que contiene mínimas cantidades de azufre y metales pesados. Los resultados mostraron que con dosificaciones de 1 a $5 \%$ en hidrocarburos pesados y extrapesados se alcanzan reducciones de la viscosidad de 32 a $74 \%$, de modo que la disminución en las fuerzas no polares son los principales cambios en las interacciones intermoleculares de las mezclas realizadas. Continuando con esta recopilación de evaluaciones de compuestos con potencial viscorreductor, Hashmi y colaboradores realizaron un estudio sobre el dispersante de asfaltenos ácido dodecil benceno sulfónico (DBSA), el cual puede estabilizar los coloides de asfalteno y disolver los asfaltenos a escala molecular de forma simultánea, valiéndose de las interacciones ácido-base $n-\pi^{*}$ entre el ácido sulfónico y los heteroátomos de los asfaltenos [62]. Respecto a ese mismo compuesto, Yang realizó la evaluación del DBSA y amina láurica (LA) sobre el estado y reología de crudos pesados [58]. Se confirmó la capacidad dispersante de asfaltenos del ácido fuerte DBSA, en virtud de un marcado aumento de la conductividad eléctrica y de la posibilidad de realizar puentes de hidrógeno e interacciones ácido-base con los asfaltenos. A pesar del comprobado efecto dispersante, la adición del DBSA aumentó la viscosidad del crudo pesado, mientras que, adversamente, la adición de LA registró una disminución de la conductividad eléctrica, lo cual implica que LA flocula las partículas de asfalteno originales en complejos más grandes, lo cual, contrariamente a lo esperado, reflejó una disminución de la viscosidad.

El uso de un tensoactivo tipo catiónico como el cloruro de dodeciltrimetilamonio (C12TAC) se menciona como dispersante de asfaltenos de un crudo mexicano ( $15^{\circ} \mathrm{API}, 22,85 \%$ asfaltenos y baja acidez) [85]. El aditivo se evaluó con cloroformo, como vehículo, y con una concentración de $0,2 \mathrm{~g} / \mathrm{L}$, esto dio como resultado reducciones de alrededor de 100-150 cP. Los autores asumen que el efecto viscorreductor se debe a la capacidad del compuesto de formar complejos supramoleculares con las resinas y asfaltenos, lo que permite la desagregación y promueve la formación de micelas entre dispersante-asfalteno. Ahora bien, desde el punto de vista del carácter electrostático del compuesto, Eddine y colaboradores evaluaron a bajas dosificaciones (10-300 ppm) un tensoactivo 
aniónico como el dodecilbencensulfonato de sodio (SDBS) en un crudo liviano de Algeria $\left(32,7^{\circ} \mathrm{API}\right)$, y de ello se obtuvo una positiva reducción de la viscosidad, la cual, aducen, depende en gran medida de la concentración del tensoactivo [21]. De otro lado, se reportó la síntesis de un terpolímero [91], que mostró una eficiencia de reducción de viscosidad de $95 \%$ a $50{ }^{\circ} \mathrm{C}$ en una concentración del $2 \%$ en un crudo pesado [21]. Los autores concluyen que el aditivo altera la estructura original intermolecular del crudo, debilitando su habilidad de formar puentes de hidrógeno con grupos carboxilos o hidroxilos. Como resultado se obtiene una disminución de la viscosidad. En otra investigación, se reportó el uso de n-aryl amino alcoholes como reductores de viscosidad en un crudo $\mathrm{Ku}$ de $21^{\circ} \mathrm{API}$, dada su capacidad de realizar interacciones con los asfaltenos tipo п- $\pi$ stacking y puentes de hidrógeno [74]. Los resultados evaluados en una matriz de n-heptano, muestran una eficiencia dispersante de hasta un $95 \%$ (concentraciones hasta $0,1 \mathrm{~g} / \mathrm{L}$ ), y además reducciones de la viscosidad de hasta un $40 \mathrm{cP}$.

Es positivo mencionar algunos compuestos con capacidad dispersante comprobada, pero que hasta el momento no se han hallado reportes concretos de los efectos en la viscosidad; tal es el caso de la síntesis y evaluación del compuesto N,N,N'trimetil-N'-octadecil-1,2-diaminometano (TODE) en asfaltenos de crudo Ayacucho $\left(10^{\circ}\right.$ API y $10 \%$ $\mathrm{m} / \mathrm{v}$ asfaltenos) [56]. Se reporta que la presencia del TODE estabilizó el asfalteno en disolución, con una efectividad muy cercana a una resina natural. Los autores atribuyen el carácter dispersante del TODE a la parte polar de dos grupos amino presentes en el compuesto, que interactúan mejor con el asfalteno y a la gran cantidad de grupos etoxilados, los cuales disminuyen la actividad dispersante. Otro ingrediente activo potencial, mencionado en la literatura, es el ácido salicílico, del cual se reportó que con una dosificación de 250 ppm y gracias a tener un grupo hidroxilo extra en el anillo benzoico, se alcanza un $34 \%$ de reducción en la precipitación de asfaltenos. Esto se asocia a la habilidad de estabilizar los asfaltenos mediante interacciones $\pi-\pi$ dadas por un anillo aromático, y que se ven potenciadas por el grupo hidroxilo extra en el anillo, además de la afinidad de los grupos ácidos por los sitios básicos de los asfaltenos, y de los puentes de hidrógeno que se generan entre el compuesto-asfalteno [83].

Finalmente, cabe mencionar que la mayor parte de los estudios están enfocados en las interacciones que se logran con los asfaltenos. Esto da un protagonismo alto a estas especies, pues se estabilizan con las resinas de la matriz. Carnhan y colaboradores resaltan la importancia que tienen las resinas en la estabilización de crudos, y, como resinas extraídas del crudo Boscan logran tener mayor efecto estabilizante sobre el crudo Hamaca, superando el efecto de las resinas nativas [92]. Pero también aclaran la falta de estudios analíticos que establezcan las causas y los mecanismos por los cuales se consigue dicho efecto. Esto muestra otro tópico a contemplar a la hora de diseñar aditivos reductores de viscosidad, de los cuales las resinas moleculares pueden llegar a comportarse como aditivos, al modificarse estructuralmente.

\section{Conclusiones}

Con la ayuda de los modelos reportados, se puede obtener una mejor descripción de los fenómenos moleculares asociados con los tipos de agregaciones moleculares responsables del aumento de la viscosidad. Esto facilita la comprensión de las posibles interacciones que ocasionan dicho fenómeno. Lo anterior es de vital importancia en el diseño de aditivos reductores de viscosidad.

Otra variable a considerar en el diseño de aditivos es el tipo de resinas existentes en los crudos, ya que son compuestos que se consideran capaces de mantener en solución a los asfaltenos y estabilizarlos por peptización.

Al asumirse que la viscosidad es influenciada por el tipo de dispersiones coloidales, esta se puede disminuir si se logra penetrar la estructura micelar, así como romper los aglomerados de asfaltenos, dispersando estos compuestos orgánicos pesados. También se puede concluir que los aditivos reductores de viscosidad, generalmente, presentan una estructura molecular compuesta de una cabeza con carácter aromático, con una cola alifática que simula a las resinas. Se sugiere que estas tengan un momento dipolar pequeño y un carácter ácido a causa de la basicidad tipo Lewis de los asfaltenos.

Finalmente, se puede evidenciar en la literatura el uso de moléculas como aditivos reductores de viscosidad, cuyas características estructurales incluyen grupos funcionales como aminas, amidas, alcoholes, ácidos, entre otros; se reporta efectividad en el proceso de reducción de viscosidad, lo que da indicio de los tipos de interacciones moleculares más importantes a la hora de atacar el problema de 
alta viscosidad en crudos pesados. Cabe resaltar el poder viscorreductor del LA, el cual logra posiblemente dicho efecto gracias al nitrógeno, en comparación al DBSA, que mostró un efecto contrario en crudos pesados, probablemente por el átomo de azufre. Con este es posible realizar diversos tipos de interacciones de largo alcance, y esto desfavorecería la estabilidad del crudo. Adicionalmente, el poder reductor del BRV es llamativo, al ser un sistema de procedencia natural (poco impacto ambiental), con poca cantidad de azufre (disminución de las interacciones intermoleculares fuertes y bajo aporte de dicho heteroátomo en el crudo. Esto disminuye su efecto en tratamientos posteriores, tales como procesos catalíticos), y con características afines con el material orgánico, lo que ofrece un posible futuro bioreductor de alto impacto para la industria Oil \& Gas.

\section{Agradecimientos}

Se agradece de manera especial al convenio marco n. ${ }^{\circ}$ 5226851, Acuerdo 001, suscrito entre la Universidad Pontificia Bolivariana (UPB), Seccional Bucaramanga, y Ecopetrol, Instituto Colombiano del Petróleo (ICP), por su financiación y ayuda para la realización del presente artículo.

Se desea dar un merecido agradecimiento a la tecnóloga química Luz Karime Contreras Briceño y al químico magíster Uriel Mora Cruz por sus valiosos aportes a la realización del presente artículo.

\section{Referencias bibliográficas}

[1] Guerrero Aconcha AL, Agudelo, Vaca LE. Mejoramiento (upgrading) de crudos pesados y extrapesados por inyección de vapor sobrecalentado y catalizadores en oleoductos (tuberia) (tesis pregrado). Bucaramanga, Colombia: Universidad Industrial de Santander; 2007.

[2] Bassane JFP, Sad CMS, Neto DMC, Santos FD, Silva M, Tozzi FC, et al. Study of the effect of temperature and gas condensate addition on the viscosity of heavy oils. J Pet Sci Eng. 2016;142:163-9.

[3] Shokrlu $\mathrm{YH}$, Babadagli T. Viscosity reduction of heavy oil/bitumen using micro- and nanometal particles during aqueous and nonaqueous thermal applications. J Pet Sci Eng. 2014;119:210-20.
[4] Li Y, Gao H, Pu W, Wei B, Chen Y, Li D, et al. Viscosity profile prediction of a heavy crude oil during lifting in twodeep artesian wells. Chinese J Chem Eng. 2017;25:976-82.

[5] Yáñez E, Ramírez A, Uribe A, Castillo E, Faaij A. Unravelling the potential of energy efficiency in the Colombian oil industry. J Clean Prod. 2018;176:604-28.

[6] Alotaibi FM, González-Cortés S, Alotibi MF, Xiao T, Al-megren H, Yang G, et al. Enhancing the production of light ole fi ns from heavy crude oils: Turning challenges into opportunities. Catal Today. 2018;(October 2017):0-1.

[7] Saniere A, Hénaut I, Argillier JF. Pipeline transportation of heavy oils, a strategy, economic and technological challenger: oil and gas. Oil Gas Sci Technol IFP. 2004;59(5):45566.

[8] Owen NA, Inderwildi OR, King DA. The status of conventional world oil reserves-Hype or cause for concern? Energy Policy. 2010;38(8):47439.

[9] Yan S, Zhuo L, Zhenxue J, Qun L, Dongdong L, Zhiye G. Progress and development trend of unconventional oil and gas geological research. Pet Explor Dev. 2017;44(4):675-85.

[10] Hosseini SH, Hamed SG. A study on the future of unconventional oil development under different oil price scenarios: A system dynamics approach. Energy Policy. 2016;91:64-74.

[11] Alboudwarej H, Felix J (John), Taylor S, Badry $\mathrm{R}$, Bremner C, Brough B, et al. Highlighting Heavy Oil. Oilfield Review. 2006;18(2):38-59.

[12] Oñate-Morales, José Anibal. RodriguezNavas RF. Evaluación de las alternativas de transporte de crudo pesado por tuberías: caso aplicado al campo rubiales (tesis pregrado). Bucaramanga, Colombia: Universidad Industrial de Santander; 2012.

[13] Kumar R, Banerjee S, Mandal A, Naiya TK. Flow improvement of heavy crude oil through pipelines using surfactant extracted from soapnuts. J Pet Sci Eng. 2017;152:353-60.

[14] Afra S, Nasr-El-Din HA, Socci D, Cui Z. Green phenolic amphiphile as a viscosity modifier and asphaltenes dispersant for heavy and extra-heavy oil. Fuel. 2018;220:481-9.

[15] Cavicchio CAM, Biazussi JL, de Castro MS, Bannwart AC, Rodriguez $\mathrm{OMH}$, de Carvalho $\mathrm{CHM}$. Experimental study of viscosity effects on heavy crude oil-water core-annular flow pattern. Exp Therm Fluid Sci. 2018; 92:270-85. [16] Hein FJ. Geology of bitumen and heavy oil: An 
overview. J Pet Sci Eng. 2017;154:551-63.

[17] Patiño EJ, Martinez CA, Novoa LA, Barrero R. Colombian asphaltenes: The ionic superficial carácter and their interactions with ionic surfactants. En: Rio Oil \& Gas Expo and Conference 2014. Rio de Janeiro: Copyright 2014, Brazilian Petroleum, Gas and Biofuels Institute - IBP; 2014.

[18] Afanasjeva N, Lizcano-valbuena $\mathrm{WH}$, Aristizabal N, Mañozca I. Electrodeposición de vanadio y níquel de los asfaltenos de crudos pesados $\mathrm{V}$ and $\mathrm{Ni}$ electrochemical deposition from asphaltenes in heavy oils. Ing y Compet. 2015;17(2):9-17.

[19] Santos RG, Loh W, Bannwart AC, Trevisan OV. An overview of heavy oil properties and its recovery and transportation methods. Brazilian J Chem Eng. 2014;31(3):571-90.

[20] Husin H, Azizi A, Husna A. An overview of viscosity reducers in heavy crude oil production. En: ResearchGate, Conference: Chemeca; 2014 sep; At Perth, Australia. p. Paper No. 838.

[21]Djemiat DE, Safri A, Benmounah A, Safi B. Rheological behavior of an Algerian crude oil containing Sodium Dodecyl Benzene Sulfonate (SDBS) as a surfactant: Flow test and study in dynamic mode. J Pet Sci Eng. 2015;133:18491.

[22] Alvarez Díaz CJ, Martínez Rey R, Patiño Reyes EJ. Estudio experimental sobre la eficiencia de un tratamiento de ultrasonido en un sistema de flujo continuo para la reducción de viscosidad de crudo pesado. rev. ion. 2013;26(2):47-63.

[23] Subramanian D, Wu K, Firoozabadi A. lonic liquids as viscosity modifiers for heavy and extra-heavy crude oils. Fuel. 2015;143(December):519-26.

[24] Herrera CD. Modelo de estabilidad de asfaltenos como herramienta para predecir el daño de formación en pozos productores de petróleo con alto contenido de $\mathrm{CH} 4, \mathrm{CO} 2$ o N2 (tesis maestría). Medellín, Colombia: Universidad Nacional De Colombia; 2015.

[25] Abarca Vinueza AG. Estudio del efecto de reductores de viscosidad en crudos pesados (tesis pregrado). Quito, Ecuador: Escuela Politécnica Nacional; 2016.

[26] Camacho Briones C, Cámara Mendoza JR. Evaluación de las tecnologías aplicadas al transporte de crudo pesado en tuberías (tesis pregrado). México D. F., México: Universidad Nacional Autónoma De México; 2014.
[27]Buenrostro-Gonzalez E, Groenzin H, LiraGaleana C, Mullins OC. Asphaltenes. Energy \& Fuels. 2001;15(13):972-8.

[28] Akbarzadeh K, Hammami A, Zhang D, Allenson S, Creek J, Kabir S, et al. Asphaltenesproblematic but rich in potential. Oilfield Review. 2007;22-43.

[29] Groenzin H, Mullins OC. Molecular size and structure of asphaltenes. Pet. Sci. Technol. 2001;19:219-30.

[30]Mullins OC. Review of the molecular struction and aggregation of asphaltenes and petroleomics. SPE J. 2008;13(1):48-57.

[31]Langevin D, Argillier JF. Interfacial behavior of asphaltenes. Adv. Colloid Interface Sci. 2016;233:83-93.

[32] Mullins OC. The Asphaltenes. Annu. Rev. Anal. Chem. 2011;4(1):393-418.

[33] Coelho RR, Hovell I, De Mello Monte MB, Middea A, De Souza AL. Characterisation of aliphatic chains in vacuum residues (VRs) of asphaltenes and resins using molecular modelling and FTIR techniques. Fuel Process Technol. 2006;87(4):325-33.

[34] Groenzin H, Mullins OC, Eser S, Mathews J, Yang MG, Jones D. Molecular size of asphaltene solubility fractions. Energy and Fuels. 2003;17(2):498-503.

[35] Yen TF. Structure of petroleum asphaltene and its significance. Energy Sources. 1974;1(4):447-63.

[36] Mullins OC. The modified yen model. Energy and Fuels. 2010;24(4):2179-207.

[37] Alcazar-Vara LA, Garcia-Martinez JA, Buenrostro-Gonzalez E. Effect of asphaltenes on equilibrium and rheological properties of waxy model systems. Fuel. 2012;93:200-12.

[38] Ebrahimi M, Mousavi-Dehghani SA, Dabir B, Shahrabadi A. The effect of aromatic solvents on the onset and amount of asphaltene precipitation at reservoir conditions: Experimental and modeling studies. J. Mol. Liq. 2016;223:119-27.

[39] Garreto MSE, Mansur CRE, Lucas EF. A model system to assess the phase behavior of asphaltenes in crude oil. Fuel. 2013;113:31822.

[40] Sjöblom J, Simon S, Xu Z. Model molecules mimicking asphaltenes. Adv. Colloid Interface Sci. 2015;218:1-16.

[41]Vafaie-Sefti M, Mousavi-Dehghani SA, Mohammad-Zadeh M. A simple model for asphaltene deposition in petroleum mixtures. 
Fluid Phase Equilib. 2003;206:1-11.

[42]Zeinali Hasanvand M, Ahmadi MA, Mosayebi Behbahani R, Feyzi F. Developing grey-box model to diagnose asphaltene stability in crude oils: Application of refractive index. Petroleum. 2016;2(4):369-80.

[43] Groenzin H, Mullins OC. Asphaltene molecular size and structure. J. Phys. Chem. A. 1999;103(50):11237-45.

[44]Velásquez I, Pereira JC. Emulsiones de agua en crudo. Aspectos Generales. Rev. Ing. UC. 2015;21(3):45-54.

[45] Hortal AR, Hurtado P, Martnez-haya B, Mullins OC, Martı B. Molecular-weight distributions of coal and petroleum asphaltenes from laser desorption / ionization experiments molecular-weight distributions of coal and petroleum asphaltenes from laser desorption / ionization experiments. Energy \& Fuels. 2007;21(15):2863-8.

[46] Andreatta G, Goncalves CC, Buffin G, Bostrom N, Quintella CM, Arteaga-larios F, et al. Nanoaggregates and structure - function relations in asphaltenes. Energy \& Fuels. 2005;19(6):1282-9.

[47]Betancourt SS, Ventura GT, Pomerantz AE, Viloria O, Dubost FX, Zuo J, et al. Nanoaggregates of asphaltene in a reservoir crude oil and reservoir connectivity. Energy Fuels. 2009;23(3):1178-88.

[48]Zeng $H$, Song $Y-Q$, Johnson DL, Mullins OC. Critical nanoaggregate concentration of asphaltenes by direct-curretn (DC) electrical conductivity. Energy \& Fuels. 2009;23(3):12018.

[49] Goual L, Sedghi M, Zeng H, Mostowfi F, McFarlane R, Mullins OC. On the formation and properties of asphaltene nanoaggregates and clusters by DC-conductivity and centrifugation. Fuel. 2011;90(7):2480-90.

[50] Mostowfi F, Indo K, Mullins OC, McFarlane R. Asphaltene nanoaggregates studied by centrifugation. Energy and Fuels. 2009;23(3):1194-200.

[51] Mullins OC, Sabbah H, Eyssautier J, Pomerantz $A E$, Barré $L$, Andrews AB, et al. Advances in asphaltene science and the Yen-Mullins model. Energy and Fuels. 2012;26(7):3986-4003.

[52] Wu Q, Seifert DJ, Pomerantz AE, Mullins OC, Zare RN. Constant asphaltene molecular and nanoaggregate mass in a gravitationally segregated reservoir. Energy and Fuels. 2014;28(5):3010-5.
[53]Zuo JY, Mullins OC, Freed D, Elshahawi H, Dong C, Seifert DJ. Advances in the floryhüggins-zuo equation of state for asphaltene gradients and formation evaluation. Energy \& Fuels. 2013;27(4):1722-35.

[54] Mousavi M, Abdollahi T, Pahlavan F, Fini EH. The influence of asphaltene-resin molecular interactions on the colloidal stability of crude oil. Fuel. 2016;183:262-71.

[55] Andreatta G, Bostrom N, Mullins OC. High-Q ultrasonic determination of the critical nanoaggregate concentration of asphaltenes and the critical micelle concentration of standard surfactants. Langmuir. 2005;21(7):2728-36.

[56] Labrador-Sánchez H, Alvarado Z, Dorta R, Rizzo A. Síntesis y evaluación del N, N, N'-trimetil-N'-octadecil-1, 2-diaminoetano (TODE) como dispersante de la fracción de asfalteno. REDIP. 2014;4(1):568-82.

[57]Al-Sahhaf TA, Fahim MA, Elkilani AS. Retardation of asphaltene precipitation by addition of toluene, resins, deasphalted oil and surfactants. Fluid Phase Equilib. 2002;194:1045-57.

[58] Yang F, Li C, Yang S, Zhang Q, Xu J. Effect of dodecyl benzene sulfonic acid (DBSA) and lauric amine (LA) on the associating state and rheology of heavy oils. J. Pet. Sci. 2014;124:1926.

[59] Badger MW, Schobert HH. Viscosity reduction in extra heavy crude oils. En: ACS Division of Fuel Chemistry, Preprints. 1998. p. 461-3.

[60]Chávez-Miyauchi TE, Zamudio-Rivera LS, Barba-López V. Aromatic polyisobutylene succinimides as viscosity reducers with asphaltene dispersion capability for heavy and extra-heavy crude oils. Energy and Fuels. 2013;27(4):1994-2001.

[61] Valbuena V, Lima L De, Ranaudo M. Obtención y caracterización molecular de resinas tipo I y resinas tipo II de crudos venezolanos. Rev. Ing. UC. 2012;19(2):25-34.

[62] Hashmi SM, Firoozabadi A. Self-assembly of resins and asphaltenes facilitates asphaltene dissolution by an organic acid. J. Colloid Interface Sci. 2013;394(1):115-23.

[63] Alvarez-Ramirez F, Ramirez-Jaramillo E, Ruiz-Morales Y. Calculation of the interaction potential curve between asphaltene- asphalten, asphaltene- resin, and resin- resin systems using density functional theory. Energy \& Fuels. 2006;20(10):195-204.

[64]De León-Barreneche J, Hoyos-Madrigal 
BA, Cañas-Marín WA. Aggregation study of asphaltenes from colombian Castilla crude oil using molecular simulation. Rev. Fac. Ing. 2015;(77):25-31.

[65] Suárez Domínguez E, Betancourt Mar J, Llanos Pérez J, Nieto Villar J, Palacio Pérez A, Izquierdo Kulich E. Dimensión fractal de asfaltenos en capa delgada en presencia de un estabilizante. Rev. Cuba Quim. 2013;XXV(3):311-8.

[66] Martínez-Martín E, Acosta-Martínez L, Ramírez-Apodaca FD. Emulsificación de petróleo crudo para su trasporte por oleoductos. Ing. Investig. y Tecnol. 2016;17(3):395-403.

[67] Reyes J, Cerón-Camacho R, Martínez-Palou R, Villanueva D, Vallejo AA, Aburto J. Study of the formation and breaking of extra-heavycrudeoil-in-water emulsions-A proposed strategy for transporting extra heavy crude oils. Chem. Eng. Process Process Intensif. 2015;98:11222.

[68] Ashrafizadeh SN, Kamran M. Emulsification of heavy crude oil in water for pipeline transportation. J. Pet. Sci. Eng. 2010;71(34):205-11.

[69]Martínez-Palou R, Lourdes M De, ZapataRendón B, Mar-Juárez E, Bernal-Huicochea C, Chávez-Lopez J, Aborto J. Transportation of heavy and extra-heavy crude oil by pipeline: A review. J Pet Sci Eng. 2011;75(3-4):274-82.

[70] Hart A. A review of technologies for transporting heavy crude oil and bitumen via pipelines. J Petrel Explor Prod Technol. 2014;4:327-36.

[71] Gateau P, Hénaut I, Barré L, Argillier JF. Heavy oil dilution. Oil Gas Sci Technol. 2004;59(5):503-9.

[72] Shigemoto N, Al-Maamari RS, Jibril BY, Hirayama A. A study of the effect of gas condesate on the viscosity and storage stability of Omani heavy crude oil. Energy and Fuels. 2006;20(6):2504-8.

[73] Kanaveli I, Atzemi M, Lois E. Predicting the viscosity of diesel / biodiesel blends. Fuel. 2017;199:248-63.

[74] Chávez-Miyauchi TE, Zamudio-Rivera LS, Barba-López V, Buenrostro-Gonzalez E, Martínez-Magadán JM. N-aryl aminoalcohols as stabilizers of asphaltenes. Fuel. 2013;110:302-9.

[75] Murgich J, Rodríguez, Aray Y. Molecular recognition and molecular mechanics of micelles of some model asphaltenes and resins. Energy \& Fuels. 1996;10(1):68-76.
[76] Ashoori S, Sharifi M, Masoumi M, Mohammad Salehi M. The relationship between SARA fractions and crude oil stability. Egypt J. Pet. 2017;26(1):209-13.

[77] Östlund JA, Nydén M, Fogler HS, Holmberg K. Functional groups in fractionated asphaltenes and the adsorption of amphiphilic molecules. Colloids Surfaces A Physicochem Eng Asp. 2004;234(1-3):95-102.

[78] Song X, Dong L, Cao X, Xu Z, Wang C, Zhang $L$, et al. Dynamic interfacial tensions of p-(n-lauryl)-benzyl polyoxyethylene ether carboxybetaine solutions. J. Pet. Sci. Eng. 2014;124:27-34.

[79]Eastoe J, Tabor RF. Surfactants and Nanoscience. En: Colloidal Foundations of Nanoscience. Berti D, Palazzo G, editors. $1^{\text {st }}$ Edition. Poland: Elsevier B.V.; 2014. p. 135-57.

[80] Foley P, Kermanshahi pour A, Beach E, Zimmerman J. Derivation and synthesis of renewable surfactants. Chem. Soc. Rev. 2012;41(4):1499-518.

[81]Alomair OA, Almusallam AS. Heavy crude oil viscosity reduction and the impact of asphaltene precipitation. Energy \& Fuels. 2013;27(12):7267-76.

[82] Spiecker PM. The impact of asphaltene chemistry and solvation on emulsion and interfacial film formation (tesis doctoral). Raleigh, Estados Unidos de America: North Carolina State University; 2001.

[83] Karambeigi MA, Nikazar M, Kharrat $R$. Experimental evaluation of asphaltene inhibitors selection for standard and reservoir conditions. J. Pet. Sci. Eng. 2016;137:74-86.

[84]Hussein HQ, Mohammad SA. Viscosity reduction of sharqi baghdad heavy crude oil using different polar hydrocarbons, oxygenated solvents. Iraqi J. Chem. Pet. Eng. 2014;15(2):39-48.

[85]Al-Shafey HI, Hashem Al, Hameed RSA, Dawood EA. Studies on the influence of long chain acrylic esters co-polymers grafted with vinyl acetate as flow improver additives of crude oils. Adv. Appl. Sci. Res. 2011;2(5):47689.

[86] Jian C, Tang T, Bhattacharjee S. Probing the effect of side-chain length on the aggregation of a model asphaltene using molecular dynamics simulations. Energy \& Fuels. 2013;27:205767.

[87]Qi Y, Zakin JL. Chemical and rheological characterization of drag-reducing cationic 
surfactant systems. Ind. Eng. Chem. Res. 2002;41(25):6326-36.

[88] Wiehe IA, Liang KS. Asphaltenes, resins, and other petroleum macromolecules. Fluid Phase Equilib. 1996;117:201-10.

[89]Llanos A, González V, González JL, Flores J, Betancourt J, Suárez J. Comparación de reducción de viscosidad entre un bioreductor de viscosidad y xilenos aplicados a hidrocarburos. Fides Unitas Scientia Et Veritas. México; 2008.

[90] Pons-Jiménez M, Cartas-Rosado R, MartínezMagadán JM, Oviedo-Roa R, CisnerosDévora $\mathrm{R}$, Beltrán $\mathrm{HI}$, et al. Theoretical and experimental insights on the true impact of
C12TAC cationic surfactant in enhanced oil recovery for heavy oil carbonate reservoirs. Colloids Surfaces A Physicochem Eng. Asp. 2014;455(1):76-91.

[91]Guo J, Wang H, Chen C, Chen Y, Xie X. Synthesis and evaluation of an oil-soluble viscosity reducer for heavy oil. Pet Sci. 2010;7(4):536-40.

[92] Carnahan NF, Salager J, Anto R, Da A. Properties of resins extracted from boscan crude oil and their effect on the stability of asphaltenes in boscan and hamaca crude oils. Energy \& Fuels. 1999;(14):309-14. 